Research Paper

\title{
Combined Hemorrhagic Shock and Unilateral Common Carotid Occlusion Induces Neurological Injury in Adult Male Rats
}

\section{Chung-Ching Chio ${ }^{1}$, Chien-Chin Hsu ${ }^{2}, 3$, Yu-Feng Tian ${ }^{4}$, , Chung-Han Wang, ${ }^{6}$ Mao-Tsun Lin 6 , Ching-Ping} Chang, 6, 7, Hung-Jung Lin²,3凶

1. Division of Neurosurgery, Department of Surgery, Chi Mei Medical Center, Tainan 710, Taiwan;

2. Department of Biotechnology, Southern Taiwan University of Science and Technology, Tainan 710, Taiwan

Department of Emergency Medicine, Chi Mei Medical Center, Tainan 710, Taiwan;

Division of General Surgery, Department of Surgery, Chi Mei Medical Center, Tainan 710, Taiwan;

. Department of Health and Nutrition, Chia Nan University of Pharmacy and Science, Tainan 717, Taiwan

Department of Medical Research, Chi Mei Medical Center, Tainan 710, Taiwan;

7. The Ph.D. Program for Neural Regenerative Medicine, Taipei Medical University, Taipei 110, Taiwan

$\triangle$ Corresponding author: Hung-Jung Lin, MD., Department of Emergency Medicine, Chi Mei Medical Center, Tainan 710, Taiwan Mailing address: No.901, Zhonghua Rd., Yongkang Dist., Tainan 710, Taiwan Phone: +886-6-2812811, ext. 52657; Fax: +886-6-2832639; E-mail: 790001@mail.chimei.org.tw or hjlin52@gmail.com

(C) Ivyspring International Publisher. This is an open access article distributed under the terms of the Creative Commons Attribution (CC BY-NC) license (https://creativecommons.org/licenses/by-nc/4.0/). See http://ivyspring.com/terms for full terms and conditions.

Received: 2017.05.15; Accepted: 2017.08.21; Published: 2017.10.15

\begin{abstract}
Background: Clinical assessment reveals that patients after surgery of cardiopulmonary bypass or coronary bypass experience postoperative cognitive dysfunction. This study aimed to investigate whether resuscitation after a hemorrhagic shock $(\mathrm{HS})$ and/or mild cerebral ischemia caused by a unilateral common carotid artery occlusion (UCCAO) can cause brain injury and concomitant neurological dysfunction, and explore the potential mechanisms.

Methods: Blood withdrawal $(6 \mathrm{~mL} / 100 \mathrm{~g}$ body weight) for $60 \mathrm{~min}$ through the right jugular vein catheter-induced an HS. Immediately after the termination of HS, we reinfused the initially shed blood volumes to restore and maintain the mean arterial blood pressure (MABP) to the original value during the 30 -min resuscitation. A cooling water blanket used to induce whole body cooling for $30 \mathrm{~min}$ after the end of resuscitation.

Results: An UCCAO caused a slight cerebral ischemia (cerebral blood flow [CBF] 70\%) without hypotension (MABP $85 \mathrm{mmHg}$ ), systemic inflammation, multiple organs injuries, or neurological injury. An HS caused a moderate cerebral ischemia (52\% of the original CBF levels), a moderate hypotension (MABP downed to $22 \mathrm{mmHg}$ ), systemic inflammation, and peripheral organs injuries. However, combined an UCCAO and an HS caused a severe cerebral ischemia ( $18 \%$ of the original CBF levels), a moderate hypotension (MABP downed to $17 \mathrm{mmHg}$ ), systemic inflammation, peripheral organs damage, and neurological injury, which can be attenuated by whole body cooling. Conclusions: When combined with an HS, an UCCAO is associated with ischemic neuronal injury in the ipsilateral hemisphere of adult rat brain, which can be attenuated by therapeutic hypothermia. A resuscitation from an HS regards as a reperfusion insult which may induce neurological injury in patients with an UCCAO disease.
\end{abstract}

Key words: hemorrhagic shock; multiple organ injuries; ischemia/reperfusion injury; hypothermia; cerebral blood flow.

\section{Introduction}

Hemorrhagic shock (HS) is regarded as the leading cause of death in trauma patients [1]. The resuscitation after an HS causes a reperfusion or reoxygenation insult [2-4], gut injury, and multiple 
organ dysfunctions [5, 6]. Clinical assessment reveals that patients after surgery of an abdominal aneurysm [7], cardiopulmonary bypass [8], or coronary bypass [9] experience postoperative cognitive dysfunction, indicating that ischemia/reperfusion injury causes brain injury. However, it is not known whether resuscitation after a [10-12] HS can cause cerebral injury and concomitant neurological dysfunction, and its potential mechanisms.

Polytrauma can be defined as a combination of multiple, simultaneous injuries to more than one body part or organ system. The combination of injuries often occurs in modern warfare and automobile and industrial accidents [13-15]. Patient outcomes following a minor polytrauma are often worse than those with a single severe injury.

In the rat, a unilateral common carotid artery occlusion (UCCAO) causes minor alterations in cerebral blood flow (CBF) in the ipsilateral hemisphere [16-19]. Combined systemic hypoxia and UCCAO induces ischemic brain damage in rats [20]. This raises the possibility that combined HS and UCCAO may also induce neurological injury.

The present study presents a new rat model of concurrent combined HS and UCCAO injury and to assess the effects of the combined injury on neurological functions. This highly reproducible model of polytrauma combined HS and brain ischemia caused by UCCAO. Hemorrhagic shock was induced by blood withdrawal for $60 \mathrm{~min}$ and followed by reinfusion of the initially shed blood volumes during the 30-min resuscitation. Unilateral ligation of the common carotid artery was performed on the right side to induce right hemisphere ischemia.

\section{Materials and Methods}

\section{Animals and ethical approval}

Adult male Sprague-Dawley rats (weight, $280 \pm 10 \mathrm{~g}$ ), were obtained from BioLASCO Taiwan Co., Ltd. All animal procedures were approved by Institutional Animal Care and Use Committee (IACUC) of Chi Mei Medical Center (IACUC no. 103121505). W housed the animals at an ambient temperature of $22 \pm 1^{\circ} \mathrm{C}$, with a $12 \mathrm{~h}$ light- dark cycle. Pellet rat chow and tap water were available ad libitum.

\section{Surgical Preparation}

All the animals were intraperitoneally with sodium pentobarbital $(40 \mathrm{mg} / \mathrm{kg})$. We cannulated a polyethylene 50 catheter (PE-50; Clay Adams, Sparks, MD, USA) into the right femoral artery for monitoring mean arterial blood pressure (MABP) with a pressure transducers attached to a Gould 4-channel polygraph (Gould, Cleveland, OH, USA) or for collecting blood samples (Figure 1). An incision was made, and the right jugular vein was cannulated with a PE-50 tube to administer shed blood. Unilateral ligation of the common carotid artery was performed on the right side to induce right hemisphere ischemia (Figure 1). Upon placing in a stereotaxic apparatus, we inserted a 24-gauge stainless-steel needle probe (diameter, 0.58 $\mathrm{mm}$; length, $40 \mathrm{~mm}$ ) into the right hippocampus using the coordinates: A, interaural $9.7 \mathrm{~mm} ; \mathrm{L}, 2.0 \mathrm{~mm}$ from the midline; and $\mathrm{H}, 4.5 \mathrm{~mm}$ from the top of the skull [21] for monitoring cerebral blood flow (CBF). We also measured the local CBF in the hippocampus with a Laserflo BPM2 laser Doppler flowmeter (Vasametics, St. Paul, NM).

\section{Controlled hemorrhage model}

Hemorrhagic shock (HS) was induced according to the methods of a previous study [22] with minor modifications. After performing baseline measurements, HS was induced by blood withdrawal (6 mL/ 100g body weight) at steady for 60 minutes through the right jugular vein catheter (Figure 1). Immediately after the termination of shock, the initially shed blood volumes were reinfused to restore and maintain the MABP to the original value during the 30-min resuscitation. Sham-shock animals underwent cannulation of the femoral artery and femoral vein followed by a laparotomy. However, no blood was withdrawn, and the MABP was kept within normal limits. Body core temperature (Tco) was continuously monitored and maintained at $37 \pm 0.5^{\circ} \mathrm{C}$ in rat groups without whole body cooling (WBC) using a $36^{\circ} \mathrm{C}$ water circulating blanket. However, in rat groups with WBC, WBC was accomplished by a cooling $16^{\circ} \mathrm{C}$ water circulating blanket for $30 \mathrm{~min}$. After the $30 \mathrm{~min}$ cooling period, the catheters were removed, femoral artery and vein were ligated, and the skin closed. Animals were returned to the normal room temperature $\left(26^{\circ} \mathrm{C}\right)$ and appropriately fed and hydrated. Rats that survived to day 3 of hemorrhage shock were considered survivors, and the data were used for analysis of the results. Blood samples were obtained from survivors on day 3 and rats were killed with sodium pentobarbital overdose and perfused via the left cardiac ventricle with normal saline followed by $10 \%$ neutral-buffered formalin.

\section{Experimental groups}

Animals were randomly divided into one control group and four experimental groups $(n=12$ per group). Experimental group rats included (i) intact rats received hemorrhage shock (HS) only (UCCAO- + $\mathrm{HS}^{+}+\mathrm{WBC}^{-}$); (ii) intact rats received both $\mathrm{HS}$ and $\mathrm{WBC}\left(\mathrm{UCCAO}^{-}+\mathrm{HS}^{+}+\mathrm{WBC}^{+}\right.$); (iii) $\mathrm{UCCAO}$ rats 
without HS; ( $\mathrm{UCCAO}^{+}+\mathrm{HS}^{-}+\mathrm{WBC}^{-}$); (iv) $\mathrm{UCCAO}^{-}$rats received HS only $\left(\mathrm{UCCAO}^{+}+\mathrm{HS}^{+}+\mathrm{WBC}^{-}\right)$; and (v) $\mathrm{UCCAO}^{+}$rats received both $\mathrm{HS}$ and $\mathrm{WBC}\left(\mathrm{UCCAO}^{+}+\right.$ $\left.\mathrm{HS}^{+}+\mathrm{WBC}^{+}\right)$. One group of rats without an UCCAO, an HS, and a WBC served as control (UCCAO-+HS-+WBC-).

\section{Neurological and motor function evaluation}

The acute neurological deficit was evaluated in all group rats the day before and three days after HS using a modified neurological severity score [mNSS] [23]. The higher the score is, the more severe the injury is, with a maximum of 14 points. Limb motor function was determined by using an inclined plane system with a microcontroller [24]. The lower the angle of the inclined plane is, the lesser the limb motor function is, with a maximum of 60 degrees.

\section{Cerebral infarction assessment}

We subjected the anesthetized animals to transcardial perfusion of heparinized $0.05 \mathrm{~mol} / \mathrm{L}$ phosphate buffered saline (PBS), followed by ice-cold $15 \%$ sucrose in PBS. We rapidly removed the brain tissues, frozen them in liquid nitrogen and then sectioned them into slices. Brain slices were stained with 2,3,5-triphenyl tetrazolium chloride (TTC) as described previously [25]. The infarct volume $\left(\mathrm{mm}^{3}\right)$, as revealed by negative TTC stains indicating dehydrogenase-deficient tissue, was measured in each slice and summed using computerized planimetry. The distortion of infarct volume caused

(A)

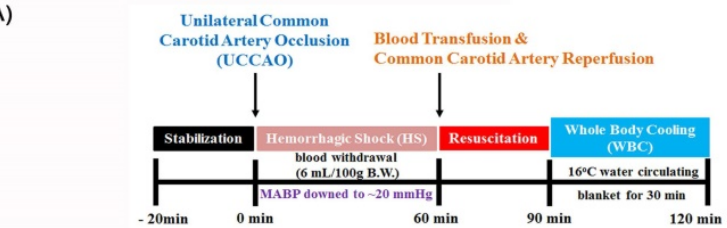

(B)

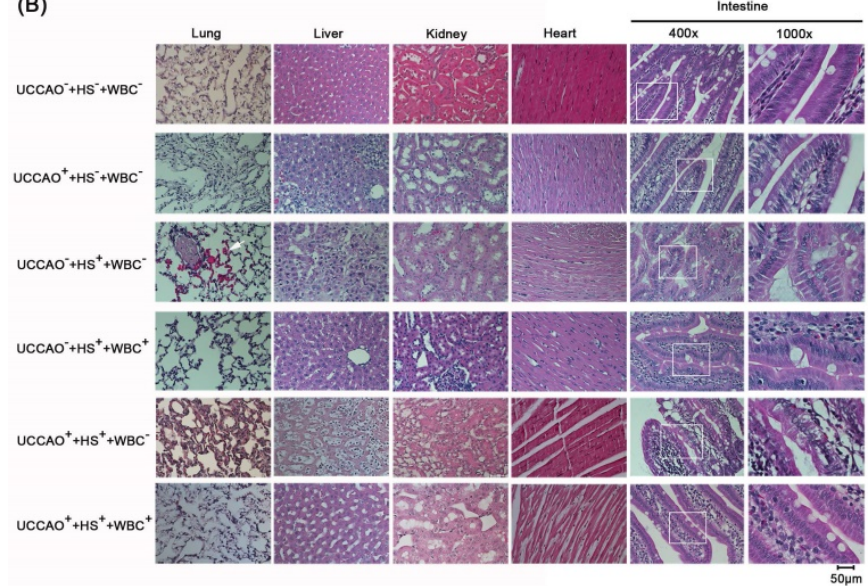

by brain edema was also corrected according to the report of Lin et al. [26].

\section{Pathohistological study}

After hematoxylin and eosin staining, a pathologist blinded for the sample grouping scored the histological changes observed in the slices. According to the descriptions detailed previously for lungs [27], intestines [28], brains [29], kidneys and livers [30], we evaluated the degrees of tissue damage.

\section{Biochemical analysis}

To assess both the renal and the hepatic functions, we determined the plasma concentrations of blood urea nitrogen (BUN), creatinine $(\mathrm{Cr})$, aspartate aminotransferase (AST), alanine aminotransferase (ALT), alkaline phosphatase (ALP), and lactate dehydrogenase (LDH) [31]. The collected plasma samples were analyzed by a chemistry analyzer (Roche Reflotron Chemistry Analyzer, Roche Diagnostic Corp., Indianapolis, IN, USA).

\section{Determination of cytokines in the serum}

The concentrations of tumor necrosis factor- $\alpha$ $(\mathrm{TNF}-\alpha)$, interleukin-1 $\beta$ (IL-1 $\beta$ ), and interleukin-6 (IL-6) in the serum were measured by enzyme-linked immunosorbent assay (ELISA) kits in accordance with the manufacturer's instructions. Serum levels of TNF- $\alpha$, IL- $1 \beta$, and IL- 6 were determined using ELISA assay kits (R \& D system, Abingdon, UK).
(C)

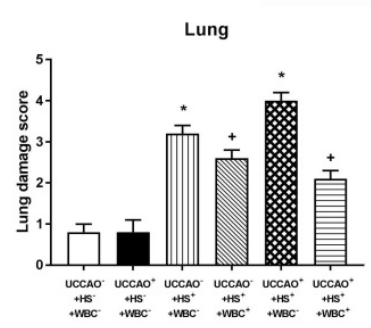

(E)

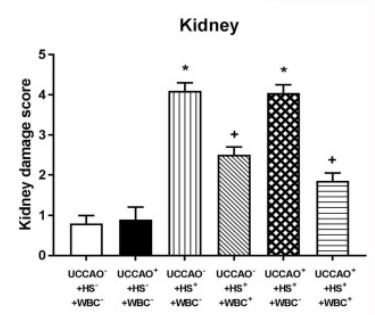

(D)

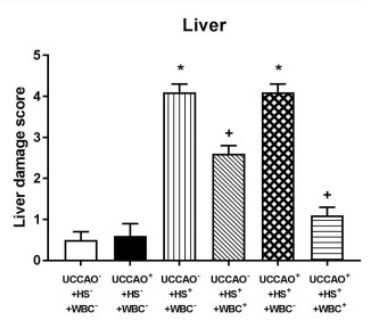

(F)

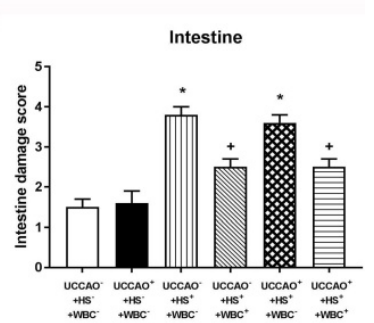

Figure 1. (A) Experimental procedures. (B-F) Representative photomicrographs of $\mathrm{H} \& \mathrm{E}$ stained slices illustrating the extent of the tissue damage obtained 3 days

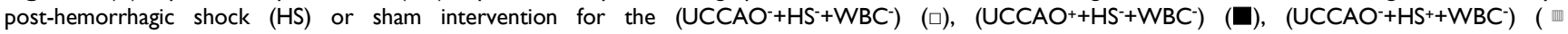

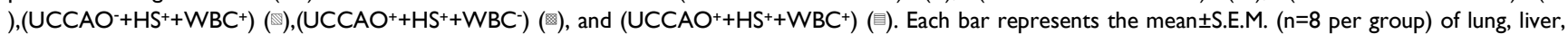
kidney, and intestine damage scores for different groups of rats. ${ }^{*}<0.05$, the (UCCAO-+HS++WBC-) vs the (UCCAO+HS+WBC-) group or the

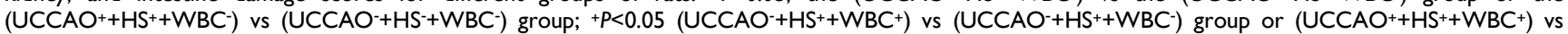
$\left(\mathrm{UCCAO}^{-}+\mathrm{HS}^{+}+\mathrm{WBC}^{-}\right)$group. 400x. Please see the explanation in the Methods section for the group abbreviations. 


\section{Immunofluorescence staining}

The brain was sliced transversely in $4 \mathrm{~mm}$ thickness, and frozen embedded. The brain slices were detected with neurons. That were covered with the mounting medium containing 4',6-diamidino-2phenylindole (DAPI; Sigma-Aldrich, St. Louis, MO, USA) for detection of total nuclei, and covered with neuronal nuclei marker(NeuN) 1:100 (Millipore Corporation, Billerica, MA, USA), second antibody anti-goat $488 \mathrm{~mm}$ (Invitrogen Life Technologies, Rockford, IL, USA), anti-mouse $588 \mathrm{~mm}$ (Invitrogen Life Technologies, Rockford, IL, USA). The other procedures for detecting apoptotic cells were performed by using a terminal deoxynucleotidyl transferase dUTP nick end labeling (TUNEL) kit (Clontech, Palo Alto, CA, USA). The slides were covered with the neuronal antibody for detection of the total neuron. Tissue pieces were checked with Carl Zeiss upright fluorescence microscope connected to an Axiocam512 camera, and the micrographs were finally analyzed with Carl Zeiss AxioVision version 4.8 software (Carl Zeiss, Jena, Germany) after staining. At brain regions, the numbers of NeuN-TUNEL-DAPI positive cells were calculated [25].

\section{Statistical analysis}

Data are presented as mean \pm S.E.M. Repeated measures analysis of variance was used to compare serial biochemical data and vital signs. We performed Chi-Square test with Fisher's exact test to analyze the survival rate. For behavioral parameters, the data were analyzed by two-way repeated factor analysis of variance. When the analysis of variance showed significance, Student- Newman-Keul's post hoc test was used. Histological scores were analyzed using Mann-Whitney U-test. The statistical software SigmaPlot 12.0 for Windows was used. A p-value $<0.05$ was considered statistically significant.

\section{Results}

\section{Combined An HS and An UCCAO Cause A More Severe Brain Ischemia}

As shown in TABLE 1, one minute before an HS or a sham intervention, all of the 6 groups of rats displayed normal levels of various physiological data including both MABP and CBF. However, 60 minutes after an HS or a sham intervention, compared to those of the (UCCAO-HS-WBC-) group rats, the $\left(\mathrm{UCCAO}^{+}+\mathrm{HS}^{-}+\mathrm{WBC}^{-}\right)$group rats had a slight cerebral ischemia $(70 \%$ vs. $93 \%$ CBF) with an insignificant hypotension $(82 \mathrm{mmHg}$ vs. $85 \mathrm{mmHg}$ MABP). The (UCCAO-+HS + WBC-) groups rats had a moderate cerebral ischemia ( $52 \%$ of the original CBF levels) and a significant hypotension (MABP downed to $22 \mathrm{mmHg}$ ). In addition, the (UCCAO $\left.{ }^{+}+\mathrm{HS}^{+}+\mathrm{WBC}^{-}\right)$ groups had a severe cerebral ischemia $(18 \%$ of the original CBF levels) and a significant hypotension (MABP downed to $17 \mathrm{mmHg}$ ). Results revealed that $\mathrm{UCCAO}^{+}$or $\mathrm{HS}^{+}$alone caused a slight to a moderate cerebral ischemia, whereas combined $\mathrm{UCCAO}^{+}$and $\mathrm{HS}^{+}$did induce a severe cerebral ischemia. Immediately after the termination of an HS, the initial

Table 1. Physiologic variables during hemorrhagic shock

\begin{tabular}{|c|c|c|c|c|c|c|}
\hline Variables & $\mathrm{UCCAO}^{-+}+\mathrm{HS}^{-}+\mathrm{WBC}-$ & $\mathrm{UCCAO}^{+}+\mathrm{HS}^{-}+\mathrm{WBC}^{-}$ & 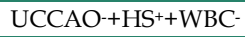 & 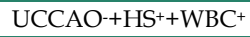 & $\mathrm{UCCAO}^{++} \mathrm{HS}^{++} \mathrm{WBC}^{-}$ & $\mathrm{UCCAO}^{+}+\mathrm{HS}^{+}+\mathrm{WBC}^{+}$ \\
\hline \multicolumn{7}{|c|}{1 min before HS/Sham intervention } \\
\hline Tco $\left({ }^{\circ} \mathrm{C}\right)$ & $36.2 \pm 0.5$ & $36.3 \pm 0.4$ & $36.3 \pm 0.4$ & $36.4 \pm 0.8$ & $36.4 \pm 0.6$ & $36.3 \pm 0.4$ \\
\hline $\mathrm{Tb}\left({ }^{\circ} \mathrm{C}\right)$ & $36.1 \pm 0.4$ & $36.0 \pm 0.3$ & $36.4 \pm 0.5$ & $36.5 \pm 0.7$ & $36.3 \pm 0.5$ & $36.5 \pm 0.3$ \\
\hline $\mathrm{MABP}(\mathrm{mmHg})$ & $86.0 \pm 3.0$ & $81.0 \pm 4.0$ & $79.0 \pm 5.0$ & $89.0 \pm 7.0$ & $83.0 \pm 3.0$ & $83.0 \pm 2.0$ \\
\hline $\mathrm{CBF}(\%)$ & $98.0 \pm 4.0$ & $97.0 \pm 5.0$ & $99.0 \pm 4.0$ & $97.0 \pm 5.0$ & $93.0 \pm 6.0$ & $99.0 \pm 5.0$ \\
\hline \multicolumn{7}{|c|}{60 min after HS/sham intervention } \\
\hline Tco $\left({ }^{\circ} \mathrm{C}\right)$ & $36.4 \pm 0.4$ & $36.5 \pm 0.5$ & $36.6 \pm 0.6$ & $36.3 \pm 0.7$ & $36.6 \pm 0.6$ & $36.8 \pm 0.5$ \\
\hline $\mathrm{Tb}\left({ }^{\circ} \mathrm{C}\right)$ & $36.3 \pm 0.6$ & $36.4 \pm 0.4$ & $36.2 \pm 0.4$ & $36.0 \pm 0.8$ & $36.2 \pm 0.5$ & $36.3 \pm 0.4$ \\
\hline MABP $(\mathrm{mmHg})$ & $85.0 \pm 2.0$ & $82.0 \pm 4.0$ & $22.0 \pm 1.0^{*}$ & $18.0 \pm 3.0^{*}$ & $17.0 \pm 3.0^{*}$ & $21.0 \pm 2.0^{*}$ \\
\hline $\mathrm{CBF}(\%)$ & $93.0 \pm 5.0$ & $70.0 \pm 3.0^{*}$ & $52.0 \pm 5.0^{*}$ & $50.0 \pm 6.0^{*}$ & $18.0 \pm 4.0^{*,+}$ & $16.0 \pm 3.0^{*++}$ \\
\hline \multicolumn{7}{|c|}{90 min after HS/sham intervention } \\
\hline Tco $\left({ }^{\circ} \mathrm{C}\right)$ & $36.3 \pm 0.4$ & $36.4 \pm 0.3$ & $36.2 \pm 0.6$ & $36.5 \pm 0.8$ & $36.6 \pm 0.5$ & $36.4 \pm 0.7$ \\
\hline $\mathrm{Tb}\left({ }^{\circ} \mathrm{C}\right)$ & $36.1 \pm 0.5$ & $36.0 \pm 0.5$ & $36.5 \pm 0.4$ & $36.2 \pm 0.6$ & $36.5 \pm 0.6$ & $36.3 \pm 0.6$ \\
\hline $\mathrm{MABP}(\mathrm{mmHg})$ & $88.0 \pm 5.0$ & $86.0 \pm 6.0$ & $84.0 \pm 6.0$ & $87.0 \pm 6.0$ & $85.0 \pm 5.0$ & $87.0 \pm 6.0$ \\
\hline CBF $(\%)$ & $100.0 \pm 6.0$ & $97.0 \pm 7.0$ & $98.0 \pm 6.0$ & $102.0 \pm 9.0$ & $99.0 \pm 7.0$ & $98.0 \pm 9.0$ \\
\hline \multicolumn{7}{|c|}{120 min after HS/sham intervention } \\
\hline Tco $\left({ }^{\circ} \mathrm{C}\right)$ & $36.5 \pm 0.6$ & $36.3 \pm 0.5$ & $36.6 \pm 0.5$ & $33.5 \pm 0.8^{*}$ & $36.3 \pm 0.4$ & $33.7 \pm 0.7^{*}$ \\
\hline $\mathrm{Tb}\left({ }^{\circ} \mathrm{C}\right)$ & $36.4 \pm 0.3$ & $36.2 \pm 0.4$ & $36.3 \pm 0.6$ & $33.2 \pm 0.7^{*}$ & $36.2 \pm 0.6$ & $33.3 \pm 0.6^{*}$ \\
\hline MABP $(\mathrm{mmHg})$ & $89.0 \pm 7.0$ & $91.1 \pm 0.8$ & $87.0 \pm 7.0$ & $89.0 \pm 5.0$ & $88.0 \pm 6.0$ & $90.0 \pm 6.0$ \\
\hline CBF (\%) & $99.0 \pm 8.0$ & $93.0 \pm 0.7$ & $101.0 \pm 8.0$ & $100.0 \pm 7.0$ & $98.0 \pm 5.0$ & $97.0 \pm 7.0$ \\
\hline
\end{tabular}

$\mathrm{Tco}=$ core body temperature; $\mathrm{Tb}=$ brain temperature; $\mathrm{MABP}=$ mean arterial pressure; $\mathrm{CBF}=$ cerebral blood flow; $\mathrm{UCCAO}^{-}=$no unilateral common carotid artery occlusion; $\mathrm{HS}^{-}=$no hemorrhagic shock; $\mathrm{WBC}^{-}=$no whole body cooling; $\mathrm{HS}^{+}$hemorrhagic shock; $\mathrm{WBC}^{+}=$whole body cooling; $\mathrm{UCCAO}^{+}=$unilateral common carotid artery occlusion Data are means \pm S.D. $\left(n=12\right.$ per group). ${ }^{*} \mathrm{P}<0.05$, compared with $\left(\mathrm{UCCAO}^{-}+\mathrm{HS}^{-}+\mathrm{WBC}^{-}\right)$group rats; ${ }^{+} \mathrm{P}^{\circ} 0.05, \mathrm{compared}$ with $\left(\mathrm{UCCAO}^{-}+\mathrm{HS}^{+}+\mathrm{WBC}^{-}\right)$or $\left(\mathrm{UCCAO}^{-}+\mathrm{HS}^{+}+\right.$ $\mathrm{WBC}^{+}$) group rats. 
shed blood volumes were reinfused to restore and maintain both the MABP $(84-88 \mathrm{mmHg})$ and the CBF $(97 \%-102 \%)$ to their original value during the 30 minutes resuscitation (TABLE 1) for all group of rats studied in the present study.

\section{HS, But Not UCCAO, Induces Peripheral Organ Injury and Systemic Inflammation}

For the present study, both peripheral injury scores and severe levels of multiple organ damage markers defined the severity of peripheral organ injury. The higher values of both the peripheral organ injury score and multiple organ damage indicators denote the more severe the peripheral organ injury. Similarly, the higher serum levels of pro-inflammatory cytokines (such as TNF- $\alpha$, IL-1 $\beta$, and IL-6) denote the more severe the systemic inflammation. As shown in both Figure 1 and TABLE 2 , the $\left(\mathrm{CCAO}^{-}+\mathrm{HS}^{+}+\mathrm{WBC}^{-}\right)$group, but not the $\left(\mathrm{UCCAO}^{+}+\mathrm{HS}^{-}+\mathrm{WBC}^{-}\right)$group rats had significantly more severe the peripheral organ injury than did the (UCCAO-+HS-+WBC-) group rats. Additionally, the extents of both peripheral organ injury and systemic inflammation in the $\mathrm{UCCAO}^{++} \mathrm{HS}^{+}+\mathrm{WBC}^{-}$group rats were insignificantly different from the $\mathrm{UCCAO}^{-}+\mathrm{HS}^{+}+\mathrm{WBC}^{-}$group rats. Results reveal that $\mathrm{HS}$, but not UCCAO, induced peripheral organ injury and systemic inflammation.

\section{Combined HS and UCCAO, but Not HS or UCCAO Alone, Induces Neurological Injury}

For the present study, both neurological motor dysfunction and pathological brain changes denote the severity of neurological injury. As depicted in Figure 2, the $\left(\mathrm{UCCAO}^{+}+\mathrm{HS}^{+}+\mathrm{WBC}^{-}\right)$group rats, but not the $\left(\mathrm{UCCAO}^{+}+\mathrm{HS}^{-}+\mathrm{WBC}^{-}\right)$group rats or the (UCCAO-+HS $++\mathrm{WBC}^{-}$) group rats, had a significantly higher neurological injury than did the (UCCAO-+HS-+WBC-) group rats. Results reveal that combined HS and UCCAO, but not HS or UCCAO alone, caused neurological injury. Behavioral tests performed 3 days after an HS or an UCCAO alone revealed that neither an HS nor an UCCAO alone significantly affected both the mNSS and the maximal angle performance. However, combined an HS and an UCCAO significantly increased the mNSS scores but significantly decreased the degrees of maximal angle.

We performed TTC stainings (Figure 2), hematoxylin-eosin stainings (H-E stain, Figure 3), immunohistochemical stainings (IHC stain, Figure 3), and immunofluorescence stainings (IF stain, Figure 3) to determine the infarction volume (Figure $2 \mathrm{~A}-\mathrm{D}$ ), neurological damage score (Figure 3 B-D), and apoptotic neuronal numbers (Figure 3 E-F), respectively.

As compared to the (UCCAO-+HS-+WBC-) group rats, the ( $\left.\mathrm{UCCAO}^{+}+\mathrm{HS}^{+}+\mathrm{WBC}^{-}\right)$group rats, but not the $\left(\mathrm{UCCAO}^{+}+\mathrm{HS}^{-}+\mathrm{WBC}^{-}\right)$group rats or the $\left(\mathrm{UCCAO}^{-}+\mathrm{HS}^{+}+\mathrm{WBC}^{-}\right)$group rats had significantly higher brain infarction volumes (Figure 2), higher cortical and striatal damage score (ipsilateral hemisphere; Figure 3B-D) and higher cortical, striatal apoptotic neuron numbers (Figure 3E and 3F).

\section{Whole Body Cooling (WBC) Attenuates Multiple Organ Injury Induced By An HS And/Or An UCCAO}

As compared to the $\left(\mathrm{UCCAO}^{+}+\mathrm{HS}^{+}+\mathrm{WBC}^{-}\right)$ group rats, the $\left(\mathrm{UCCAO}^{+}+\mathrm{HS}^{+}+\mathrm{WBC}^{+}\right)$group rats had significantly lower serum levels of multiple organ injury indicators, inflammatory cytokines (TABLE 2), peripheral organ damage scores (Figure 1), lesser brain infarction volume (Figure 2), lower cortical and striatal neuron damage scores, and lesser cortical and striatal apoptotic neuron numbers (Figure 3). Results reveal that WBC attenuated multiple organs (include brain) injury caused by an HS and/or an UCCAO in rats.

Table 2. Indicators of renal, hepatic, and inflammatory function $120 \mathrm{~min}$ after hemorrhagic shock or sham intervention

\begin{tabular}{|c|c|c|c|c|c|c|}
\hline Indicators & UCCAO-+HS-+WBC- & $\mathrm{UCCAO}^{+}+\mathrm{HS}^{-}+\mathrm{WBC}^{-}$ & $\mathrm{UCCAO}^{-}+\mathrm{HS}^{+}+\mathrm{WBC}-$ & $\mathrm{UCCAO}^{-}+\mathrm{HS}^{+}+\mathrm{WBC}^{+}$ & $\mathrm{UCCAO}^{++} \mathrm{HS}^{++} \mathrm{WBC}^{-}$ & $\mathrm{UCCAO}^{+}+\mathrm{HS}^{+}+\mathrm{WBC}^{+}$ \\
\hline Creatinine $(\mathrm{mg} / \mathrm{dL})$ & $0.42 \pm 0.02$ & $0.41 \pm 0.02$ & $0.77 \pm 0.03^{*}$ & $0.45 \pm 0.01^{+}$ & $0.76 \pm 0.07^{*}$ & $0.44 \pm 0.03^{+}$ \\
\hline BUN (mg/dL) & $16.8 \pm 0.6$ & $16.2 \pm 0.50$ & $33 \pm 5^{*}$ & $24 \pm 4^{+}$ & $34 \pm 3^{*}$ & $17 \pm 2^{+}$ \\
\hline AST (U/L) & $99 \pm 8$ & $97 \pm 7$ & $334 \pm 78^{*}$ & $210 \pm 52^{+}$ & $327 \pm 15^{*}$ & $90 \pm 6^{+}$ \\
\hline ALT (U/L) & $61 \pm 6$ & $59 \pm 5$ & $189 \pm 19^{*}$ & $97 \pm 8^{+}$ & $202 \pm 24^{*}$ & $85 \pm 5^{+}$ \\
\hline $\operatorname{ALP}(\mathrm{U} / \mathrm{L})$ & $178 \pm 66$ & $171 \pm 59$ & $647 \pm 92^{*}$ & $280 \pm 27^{+}$ & $724 \pm 111^{*}$ & $251 \pm 23^{+}$ \\
\hline TNF- $\alpha(\mathrm{pg} / \mathrm{ml})$ & $12 \pm 2$ & $11 \pm 2$ & $23 \pm 3^{*}$ & $15 \pm 3^{+}$ & $25 \pm 2^{*}$ & $13 \pm 1^{+}$ \\
\hline IL-1 $\beta(\mathrm{pg} / \mathrm{ml})$ & $18 \pm 3$ & $17 \pm 3$ & $26 \pm 4^{*}$ & $17 \pm 1^{+}$ & $27 \pm 4^{*}$ & $16 \pm 3^{+}$ \\
\hline IL-6 $(\mathrm{pg} / \mathrm{ml})$ & $4 \pm 1$ & $5 \pm 2$ & $15 \pm 3^{*}$ & $5 \pm 1^{+}$ & $17 \pm 3^{*}$ & $4 \pm 2^{+}$ \\
\hline
\end{tabular}

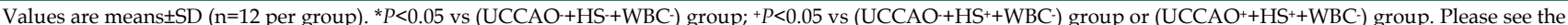
legends of TABLE 1 for explanations of group abbreviations. 
(A)

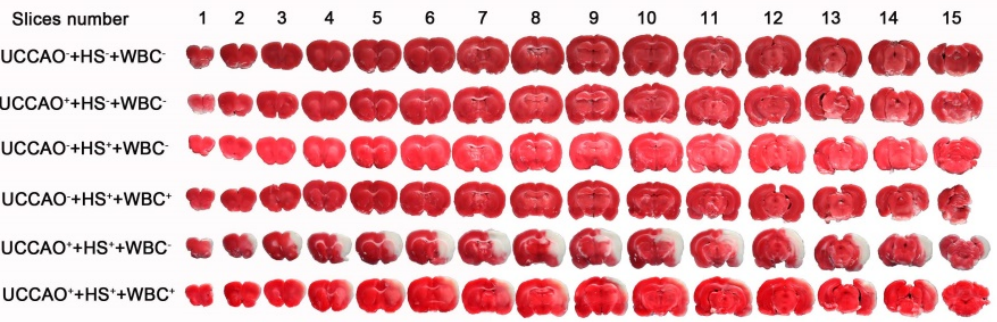
(B)

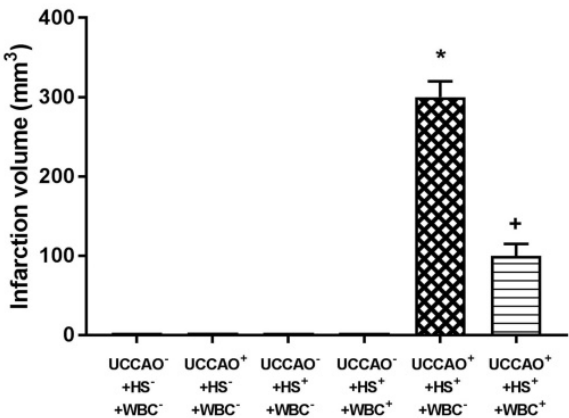

(C)

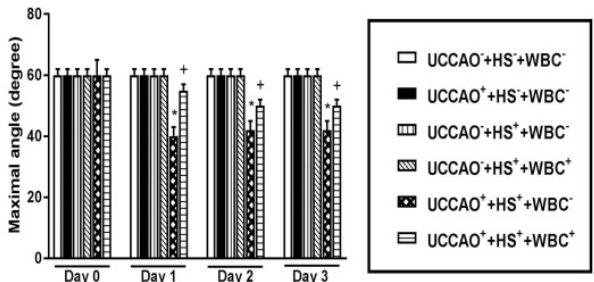

(D)

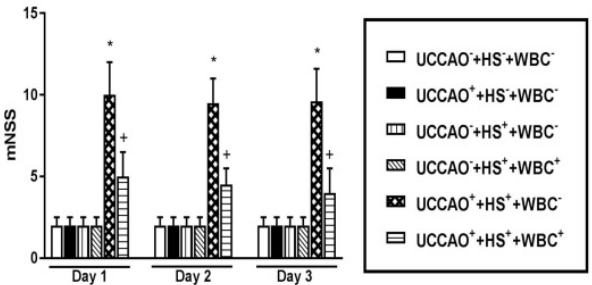

Figure 2. (A) Representative photomicrographs of TTC-stained $1 \mathrm{~mm}$ thick coronal slices illustrating the extent of the cerebral infarct obtained 3 days post-hemorrhagic shock

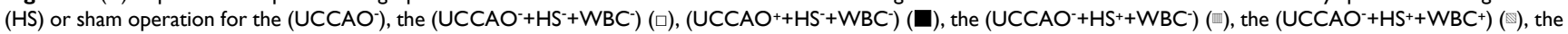

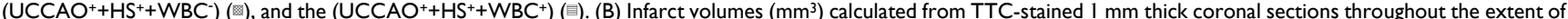
the infarct following an $\mathrm{HS}$ or a sham operation for different groups of rats. (C) maximal angle (degrees) and (D) mNSS score obtained 1 day before a hemorrhagic shock (HS) and 3 days after a $\mathrm{HS}$ for the $\left(\mathrm{UCCAO}^{-}+\mathrm{HS}^{-}+\mathrm{WBC}^{-}\right)(\square)$, the $\left(\mathrm{UCCAO}^{+}+\mathrm{HS}^{-}+\mathrm{WBC}^{-}\right)(\mathbb{\square})$ the $\left(\mathrm{UCCAO}^{-}+\mathrm{HS}^{+}+\mathrm{WBC}^{-}\right)(\mathbb{m})$, the $\left(\mathrm{UCCAO}^{-}+\mathrm{HS}^{+}+\mathrm{WBC}^{+}\right)(\mathbb{})$, the

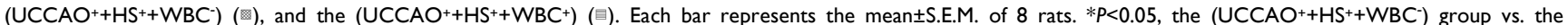
$\left(\mathrm{UCCAO}^{-}+\mathrm{HS}^{+}+\mathrm{WBC}^{-}\right)$group; $+P<0.05$, the $\left(\mathrm{UCCAO}^{+}+\mathrm{HS}^{+}+\mathrm{WBC}^{+}\right)$vs the $\left(\mathrm{UCCAO}^{+}+\mathrm{HS}^{+}+\mathrm{WBC}^{-}\right)$group.

(A) Contralateral Hemisphere

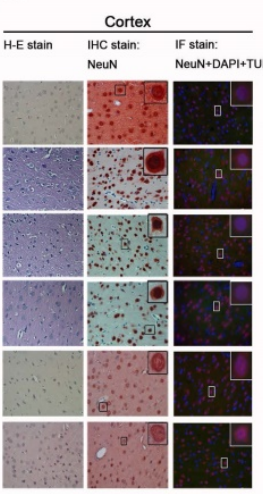

(B) Ipsilateral Hemisphere
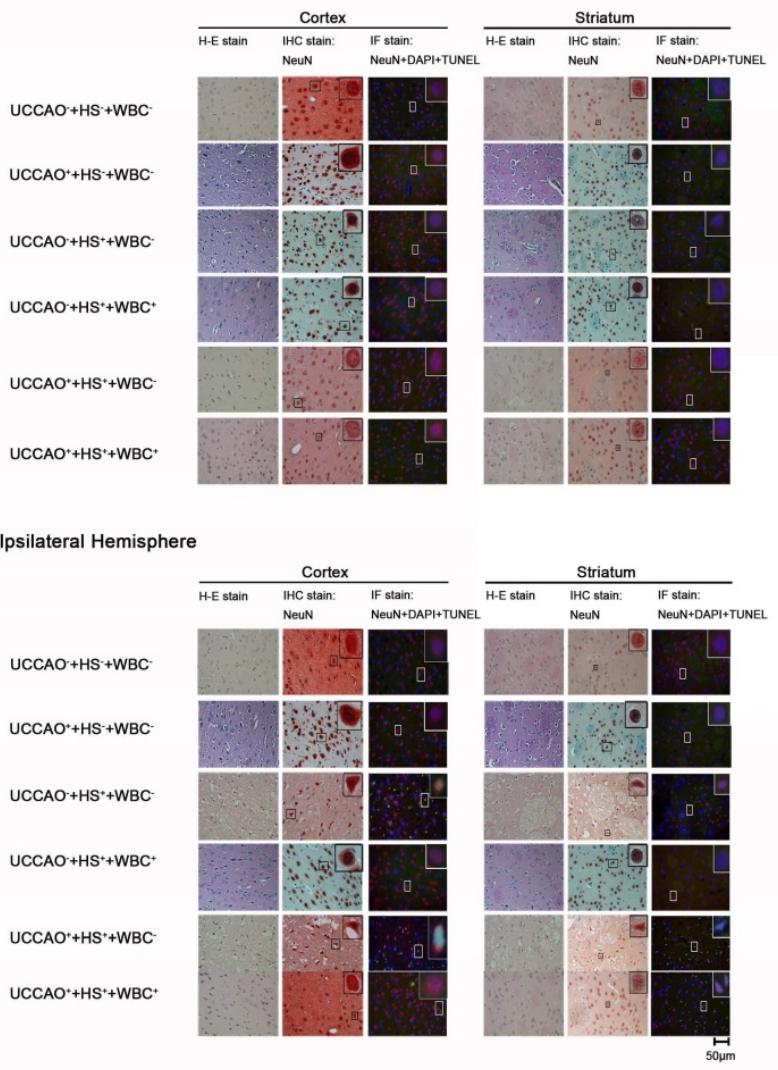

(C)

(E)
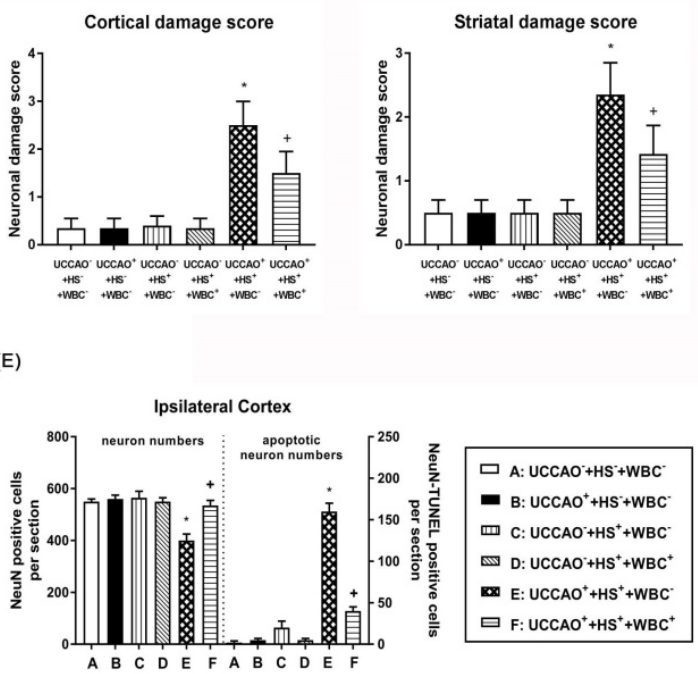

(F)

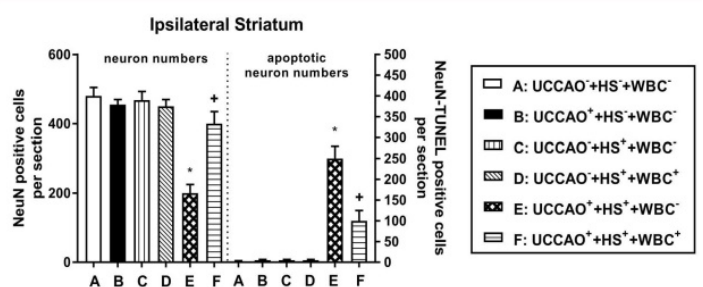

Figure 3. Representative photomicrographs of hematoxylin-eosin (HE), immunohistochemical (IHC), and NeuN-DAPI-TUNEL stainings of contralateral (A) and ipsilateral (B) cortical and striatal slices illustrating the extent of the brain injury obtained 3 days post-HS or sham intervention for the $\left(\mathrm{UCCAO}^{-}+\mathrm{HS}^{-}+\mathrm{WBC}^{-}\right)(\square)$, the $\left(\mathrm{UCCAO}^{-}+\mathrm{HS}^{-}+\mathrm{WBC}^{-}\right)$

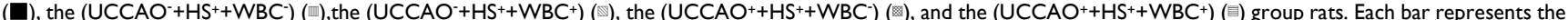
mean \pm S.E.M. ( $n=8$ per group) of cortical $(C)$ and striatal $(D)$ neuronal damage scores, numbers of NeuN positive cells and NeuN-DAPI-TUNEL positive cells in the ipsilateral cortex $(E)$ and striatum $(F)$ for different groups of rats. ${ }^{*} P<0.01$, the $\left(\mathrm{UCCAO}^{++} \mathrm{HS}^{++} \mathrm{WBC}\right)$ group vs the $\left(\mathrm{UCCAO}^{-}+\mathrm{HS}^{-}+\mathrm{WBC}^{-}\right)$group; ${ }^{+} \mathrm{P}<0.05$, the $\left(\mathrm{UCCAO}^{++} \mathrm{HS} \mathrm{S}^{++} \mathrm{WBC}^{+}\right)$ vs the (UCCAO++HS++WBC-) group. 400x. 


\section{Discussion}

Present data show that an UCCAO alone did not affect MABP $(85 \mathrm{mmHg}$ ) but caused a slight cerebral ischemia $(\sim 70 \% \mathrm{CBF})$, whereas an HS alone caused both hypotension $(22 \mathrm{mmHg}$ ) and a moderate cerebral ischemia (52\% CBF). However, combined an UCCAO and an HS caused both hypotension $(17 \mathrm{mmHg})$ and a severe cerebral ischemia (18\% CBF). An HS, but not an UCCAO caused peripheral organs injury and systemic inflammation. Combined an UCCAO and an $\mathrm{HS}$, in addition to causing both peripheral organ injury and systemic inflammation, did induce neurological injury which could be attenuated by whole body cooling. Our results reveal that an UCCAO alone or resuscitation from an HS caused only a slight or a moderate cerebral ischemia which did not induce neurological injury. However, combined an UCCAO and an HS caused more severe cerebral ischemia and resulted in neurological injury accompanied by both systemic inflammation and peripheral organs injury.

Our present results are consistent with several previous investigations. For example, occlusion of one common carotid artery alone caused only minor cerebral ischemia in the ipsilateral hemisphere [16-18]. Combined UCCAO and systemic hypoxia-induced ischemic neuronal injury in the ipsilateral hemisphere of both adult and neonatal rat brain [20, 32, 33]. In many circumstances, patients with a traumatic brain injury suffered from multiple organ injuries [34-36].

As shown in the present study, hemorrhagic shock not only causes intestinal injury but also leads to injury of other vital organs including liver, kidney, and lung and even brain. A systemic ischemic/reperfusion injury following an HS and a subsequent resuscitation causes intestinal epithelial damage in an early event and leads to the development of multiple organ dysfunctions [37, 38]. Intestinal ischemia/reperfusion resulted in significant increases in the levels of tumor necrosis factor- $\alpha$ and interleukin- 6 both in the serum and in cortical and hippocampal regions, committed with microglial activation, a key cellular mediator in neuroinflammation and neurodegeneration [39]. Our present results demonstrated that in intact (UCCAO-) rats with reduction of $\sim 50 \%$ of $\mathrm{CBF}$, overproduction of TNF- $\alpha$, IL-1 $\beta$, and IL- 6 in the peripheral blood stream alone did not cause neuronal cell apoptosis during an $\mathrm{HS}$. In contrast, in $\mathrm{UCCAO}^{+}$rats with reduction of $\sim 20 \%$ of CBF, profound cerebral ischemia (or $\sim 18 \%$ of CBF) may disrupt the blood-brain-barrier and allow more pro-inflammatory cytokine to enter into the brain. Microglial activation induced by pro-inflammatory cytokines causes neuronal cell apoptosis [40, 41]. Cells or tissues injured by an HS may recover following a resuscitation or reperfusion. However, resuscitation or reperfusion may exacerbate multiple organ injuries by the generation of reactive oxygen species and activation of destructuring enzymatic reactions [42]. Our present data further demonstrated that hypothermia attenuated the neurological injury after resuscitation from an HS by reducing cerebral ischemia.

Indeed, mild hypothermia $\left(32-34^{\circ} \mathrm{C}\right)$ has been indicated to prevent reperfusion injury after cerebral or visceral ischemia caused by a circulatory arrest [43-45]. Therapeutic hypothermia may exert its beneficial effects mainly via reduction of metabolic rate which leads to preservation of tissue energy state and intracellular $\mathrm{pH}$ in both heart and brain [46]. Hypothermia reduces resuscitation fluid volumes required to maintain blood pressure in a rat HS model [22]. Hypothermia improves the outcome of an HS by reducing reactive oxygen species expression as well as microvascular permeability [47]. Our present data further suggest that hypothermia improves results of HS by maintaining a normal CBF in an HS model. Hypothermia during resuscitation significantly ameliorates multiple organs (including brain) injury in HS rats.

\section{Conclusion}

An UCCAO alone or an HS alone caused only a slight $(70 \% \mathrm{CBF})$ or a moderate $(52 \% \mathrm{CBF})$ cerebral ischemia and did not cause neurological injury. A severe cerebral ischemia (or cerebral blood flow denoted to $17 \%$ of the original level) following a combined HS and UCCAO caused neurological injury. In this study, we provide the first data to show that a resuscitation after an HS is regarded as a reperfusion insult which may induce neurological injury in patients with the carotid occlusive disease. This information is immediately clinically applicable for critical care physicians and emergency physicians treating such patients.

\section{Abbreviations}

HS: hemorrhagic shock

UCCAO: unilateral common carotid artery occlusion

MABP: mean arterial blood pressure

Tco: core temperature

Tb: brain temperature

WBC: whole body cooling

mNSS: neurological severity score

TTC: 2,3,5-triphenyl tetrazolium chloride

BUN: blood urea nitrogen

Cr: creatinine

AST: aspartate aminotransferase

ALT: alanine aminotransferase 
ALP: alkaline phosphatase

LDH: lactate dehydrogenase

IL-1 $\beta$ : interleukin-1 beta

IL-6: interleukin-6

TNF- $\alpha$ : tumor necrosis factor-alpha

NeuN: neuronal nuclei

TUNEL: terminal deoxynucleotidyl transferase dUTP

nick end labeling

DAPI: 4',6-diamidino-2-phenylindole

\section{Acknowledgements}

This study was supported by grants from the Ministry of Science and Technology (Taipei, Taiwan) (MOST 104-2314-B-384-003-MY3, MOST 104-2320-B384-002) and Chi Mei Medical Center (Tainan, Taiwan) grant CMFHT 10504.

\section{Author Contributions}

C.H.W and C.P.C performed the animal experiments and obtained the in vivo data. H.J.L, M.T.L, and C.C.C designed the study and wrote the manuscript. Y.F.T and C.P.C analysis the data. C.C.C, and C.P.C obtained the funding for the study.

\section{Competing Interests}

The authors have declared that no competing interest exists.

\section{References}

1. Angele MK, Schneider $\mathrm{CP}$, Chaudry IH. Bench-to-bedside review: latest results in hemorrhagic shock. Crit Care. 2008; 12: 218

2. Douzinas EE, Livaditi $\mathrm{O}$, Andrianakis $\mathrm{I}$, et al. The effect of hypoxemic resuscitation from hemorrhagic shock on blood pressure restoration and on oxidative and inflammatory responses. Intensive Care Med. 2008; 34: 1133-41.

3. Kapoor R, Kalra J, Prasad K. Cardiac depression and cellular injury in hemorrhagic shock and reinfusion: role of free radicals. Mol Cell Biochem. 1997; 176: 291-301.

4. Kapoor R, Prasad K. Role of oxyradicals in cardiovascular depression and cellular injury in hemorrhagic shock and reinfusion: effect of SOD and catalase Circ Shock. 1994; 43: 79-94.

5. Ciesla DJ, Moore EE, Johnson JL, et al. The role of the lung in postinjury multiple organ failure. Surgery. 2005; 138: 749-57; discussion 57-8

6. Clark JA, Coopersmith CM. Intestinal crosstalk: a new paradigm for understanding the gut as the "motor" of critical illness. Shock. 2007; 28: 384-93.

7. Benoit AG, Campbell BI, Tanner JR, et al. Risk factors and prevalence of perioperative cognitive dysfunction in abdominal aneurysm patients. J Vasc Surg. 2005; 42: 884-90.

8. Ramlawi B, Rudolph JL, Mieno S, et al. Serologic markers of brain injury and cognitive function after cardiopulmonary bypass. Ann Surg. 2006; 244 : 593-601.

9. Djaiani G, Fedorko L, Borger MA, et al. Continuous-flow cell saver reduces cognitive decline in elderly patients after coronary bypass surgery. Circulation. 2007; 116: 1888-95.

10. Butcher NE, D'Este C, Balogh ZJ. The quest for a universal definition of polytrauma: a trauma registry-based validation study. J Trauma Acute Care Surg. 2014; 77: 620-3.

11. Paffrath T, Lefering R, Flohe S. How to define severely injured patients? -- an Injury Severity Score (ISS) based approach alone is not sufficient. Injury. 2014; 45 Suppl 3: S64-9.

12. Butcher N, Balogh ZJ. The definition of polytrauma: the need for international consensus. Injury. 2009; 40 Suppl 4: S12-22.

13. Atiyeh BS, Gunn SW, Hayek SN. Military and civilian burn injuries during armed conflicts. Ann Burns Fire Disasters. 2007; 20: 203-15.

14. Owens BD, Kragh JF, Jr., Wenke JC, et al. Combat wounds in operation Iraqi Freedom and operation Enduring Freedom. J Trauma. 2008; 64: 295-9.

15. Santaniello JM, Luchette FA, Esposito TJ, et al. Ten year experience of burn, trauma, and combined burn/trauma injuries comparing outcomes. J Trauma. 2004; 57: 696-700; discussion 00-1.
16. Coyle P, Panzenbeck MJ. Collateral development after carotid artery occlusion in Fischer 344 rats. Stroke. 1990; 21: 316-21.

17. De Ley G, Nshimyumuremyi JB, Leusen I. Hemispheric blood flow in the rat after unilateral common carotid occlusion: evolution with time. Stroke. 1985; 16: 69-73.

18. Salford LG, Siesjo BK. The influence of arterial hypoxia and unilateral carotid artery occlusion upon regional blood flow and metabolism in the rat brain. Acta Physiol Scand. 1974; 92: 130-41.

19. Bronner G, Mitchell K, Welsh FA. Cerebrovascular adaptation after unilateral carotid artery ligation in the rat: preservation of blood flow and ATP during forebrain ischemia. J Cereb Blood Flow Metab. 1998; 18: 118-21.

20. Rice JE 3rd, Vannucci RC, Brierley JB. The influence of immaturity on hypoxic-ischemic brain damage in the rat. Ann Neurol. 1981; 9: 131-41.

21. Paxinos G, Watson C. The rat brain in stereotoxic coordinates; 2nd ed. New York: Academic Press. 1982.

22. Nishi K, Takasu A, Shibata M, et al. Hypothermia reduces resuscitation fluid volumes required to maintain blood pressure in a rat hemorrhagic shock model. J Trauma Acute Care Surg. 2012; 72: 130-5.

23. Shohami E, Bass R, Wallach D, et al. Inhibition of tumor necrosis factor alpha (TNFalpha) activity in rat brain is associated with cerebroprotection after closed head injury. J Cereb Blood Flow Metab. 1996; 16: 378-84.

24. Chang MW, Young MS, Lin MT. An inclined plane system with microcontroller to determine limb motor function of laboratory animals. J Neurosci Methods. 2008; 168: 186-94.

25. Bederson JB, Pitts LH, Germano SM, et al. Evaluation of 2,3,5-triphenyltetrazolium chloride as a stain for detection and quantification of experimental cerebral infarction in rats. Stroke. 1986; 17: 1304-8.

26. Lin TN, He $Y Y, W u$ G, et al. Effect of brain edema on infarct volume in a focal cerebral ischemia model in rats. Stroke. 1993; 24: 117-21.

27. Deree J, Martins J, de Campos T, et al. Pentoxifylline attenuates lung injury and modulates transcription factor activity in hemorrhagic shock. J Surg Res. 2007: 143: 99-108.

28. Zhao B, Fei J, Chen Y, et al. Pharmacological preconditioning with vitamin C attenuates intestinal injury via the induction of heme oxygenase-1 after hemorrhagic shock in rats. PLoS One. 2014; 9: e99134.

29. Gilat E, Kadar T, Levy A, et al. Anticonvulsant treatment of sarin-induced seizures with nasal midazolam: an electrographic, behavioral, and histological study in freely moving rats. Toxicol Appl Pharmacol. 2005; 209: 74-85.

30. Chang KY, Tsai PS, Huang TY, et al. HO-1 mediates the effects of HBO pretreatment against sepsis. J Surg Res. 2006; 136: 143-53.

31. Kengatharan KM, De Kimpe SJ, Thiemermann C. Role of nitric oxide in the circulatory failure and organ injury in a rodent model of gram-positive shock. Br J Pharmacol. 1996; 119: 1411-21.

32. Levine S. Anoxic-ischemic encephalopathy in rats. Am J Pathol. 1960; 36: 1-17.

33. Salford LG, Plum F, Brierley JB. Graded hypoxia-oligemia in rat brain. II. Neuropathological alterations and their implications. Arch Neurol. 1973; 29: 234-8.

34. Levy RM, Prince JM, Yang R, et al. Systemic inflammation and remote organ damage following bilateral femur fracture requires Toll-like receptor 4. Am J Physiol Regul Integr Comp Physiol. 2006; 291: R970-6.

35. Tsukamoto T, Pape HC. Animal models for trauma research: what are the options? Shock. 2009; 31: 3-10.

36. Vincent JL. Prevention and therapy of multiple organ failure. World J Surg. 1996; 20: 465-70.

37. Aldridge AJ. Role of the neutrophil in septic shock and the adult respiratory distress syndrome. Eur J Surg. 2002; 168: 204-14.

38. Lee WL, Downey GP. Neutrophil activation and acute lung injury. Curr Opin Crit Care. 2001: 7: 1-7.

39. Zhou J, Huang WQ $\mathrm{Li} \mathrm{C}$, et al. Intestinal ischemia/reperfusion enhances microglial activation and induces cerebral injury and memory dysfunction in rats. Crit Care Med. 2012; 40: 2438-48.

40. Chio CC, Lin MT, Chang CP. Microglial activation as a compelling target for treating acute traumatic brain injury. Curr Med Chem. 2015; 22: 759-70.

41. Gelosa P, Lecca D, Fumagalli M, et al. Microglia is a key player in the reduction of stroke damage promoted by the new antithrombotic agent ticagrelor. J Cereb Blood Flow Metab. 2014; 34: 979-88.

42. Lee JH, Kim K, Jo YH, et al. Blood pressure-targeted stepwise resuscitation for hemorrhagic shock in rats. J Trauma Acute Care Surg. 2014; 76: 771-8.

43. Takasu A, Carrillo P, Stezoski SW, et al. Mild or moderate hypothermia but not increased oxygen breathing prolongs survival during lethal uncontrolled hemorrhagic shock in rats, with monitoring of visceral dysoxia. Crit Care Med. 1999; 27: 1557-64.

44. Takasu A, Norio H, Sakamoto T, et al. Mild hypothermia prolongs the survival time during uncontrolled hemorrhagic shock in rats. Resuscitation. 2002; 54: 303-9.

45. Takasu A, Sakamoto T, Okada Y. Effect of induction rate for mild hypothermia on survival time during uncontrolled hemorrhagic shock in rats. J Trauma. 2006; 61: 1330-5.

46. Kirklin J, Barratt-Boyes B. Hypothermia, circulatory arrest, and cardiopulmonary bypass. Cardiac surgery. 1993; 1: 61-127.

47. Childs EW, Udobi KF, Hunter FA. Hypothermia reduces microvascular permeability and reactive oxygen species expression after hemorrhagic shock. J Trauma. 2005; 58: 271-7. 